\title{
TOKEN BASED AUTHENTICATION FOR HANDOVER SECURITY
}

\author{
Yi Cheng, Arne Norefors \\ Ericsson Radio Systems $A B$ \\ SE-164 80 Stockholm \\ Sweden \\ yi.cheng@era.ericsson.se \\ arne.norefors@era.ericsson.se
}

\begin{abstract}
In wireless mobile communications when a user moves from one access point to another, there is a risk that an attacker pretending to be that user will get accepted by the second access point and thus be able to hijack the communication session from the real user. To thwart this kind of attack, during handover measures must be taken to authenticate the user before granting access. In this paper we propose a security token based approach which, compared to other existing approaches, gives the best comprehensive result with respect to security assurance, processing efficiency and handover performance. We present the design of a token based authentication protocol that effectively prevents impostors from capturing the communication channel at handover. The protocol, with two alternatives, is widely applicable to various network environments, and it imposes minimal processing overhead on mobile devices. This protocol has been proposed to be one part of the security solution for the HIPERLAN Type 2 wireless local area networks.
\end{abstract}

Key words handover, authentication, token 


\section{INTRODUCTION}

Wireless communications have undergone a tremendous growth in recent years, which makes user and device mobility possible. Users carrying small devices with wireless communication capabilities can access a large pool of networked resources from anywhere at anytime. However this exciting technology introduces more security vulnerabilities compared to wired fixed networking. Due to its broadcast nature, wireless communication is vulnerable to eavesdropping. Due to user mobility, a wireless network is highly dynamic, with mobile terminals coming, changing access points, and leaving all the time. Within such a dynamic system there are more opportunities for an adversary to tap in and exploit security loopholes.

In wireless communication when a mobile terminal (MT) moves from one access point (AP) to another, a handover occurs. The first time a MT attempts to access the network via an AP, the MT is usually authenticated before being accepted. Once authenticated, the MT can migrate from AP to AP without restriction. This kind of free handover exposes the network to impostors. An impostor may monitor an authorised MT. When the MT is about to change access point, the impostor immediately requests connection to the intended AP by using that MT's identity. This AP will accept the impostor as an authorised terminal handed over by the previous AP, see Fig. 1. In this way the impostor can easily hijack the communication session and grab network resources for its own use. In addition, the real mobile may be denied service because the network rejects its "duplicate connection".

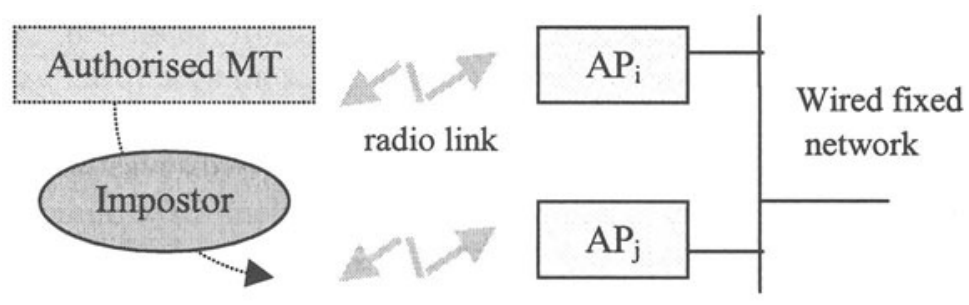

Fig 1 . Session hijacking by impostor at handover

An impostor could also, at any time, capture a communication channel by transmitting signals with higher power than the real user. This attack can be effectively countered by using channel encryption. Encryption could also be a means to ease the handover hijacking problem, but later in this paper we 
will show that encryption alone is not sufficient as handovers change one end of the communication channel.

Besides, a false AP can seduce a MT to connect to it and thus hijack the communication. If the false AP is standalone and not connected to the fixed network, it will not lead to big problems. If someone managed to set up a false AP in the wired fixed network, then the network was already penetrated. The security of fixed networks is outside the scope of this paper. From now on we only discuss the authentication of mobile terminals in the wireless environment.

It is clear that measures should be taken to authenticate handovers in a wireless mobile system. However there are some other factors that need to be taken into account. Mobile devices, such as laptops and hand-held PDAs (Personal Digital Assistants), are designed to be portable, small and light. Some of them might have only low or moderate computational power. Besides in certain circumstances (e.g. a user keeps moving) handover could occur very frequently. Therefore the overhead imposed by handover authentications should be reduced. Otherwise the overall system performance will be quite low because a large amount of time is spent on authentication rather than actual communication.

This paper presents a handover authentication protocol that is effective and lightweight. The rest of the paper is organised as follows. Section 2 gives an overview of the current approaches to handover security in existing wireless mobile systems. In Section 3 we introduce the token based approach and present our design of a specific protocol which has two alternatives to fit into different environments. Section 4 concludes the paper.

\section{CURRENT APPROACHES TO HANDOVER SECURITY}

If we refer to a set of network entities that are controlled by a single administrative authority and governed by a single security policy as a security domain, handover could be intra-domain or inter-domain. Intradomain handover occurs when a mobile terminal is moving between access points that belong to the same security domain. If a terminal crosses the domain boundary, it takes an inter-domain handover. In this case there is no doubt that the terminal should be authenticated before entering the new 
security domain. In the following sections we focus on approaches to intradomain handover authentication.

\subsection{Reuse of security associations}

As the title implies, this approach reuses established security associations after handover. When a MT first enters a security domain, it authenticates itself and establishes a security relationship to the domain. The security attributes representing the established relationship are kept for later use. Whenever an AP receives a handed-over MT, it retrieves the security attributes associated to that MT, for example, a session key. After that the AP can communicate with the MT by reusing those security attributes. The established security association remains valid until the MT leaves the current domain or switches off. Both GSM (Global System for Mobile Communications) [1] and CDPD (Cellular Digital Packet Data) [2] adopt this approach.

In GSM when a mobile station (MS) is switched on or it moves into a new location area, it notifies the local mobile switching centre (MSC) of its presence. The local visiting location register (VLR) contacts the MS's home location register (HLR) and obtains a set of triplets each of which contains a challenge (RAND), a signed response (SRES), and a corresponding session key $\left(\mathrm{K}_{\mathrm{c}}\right)$. The MS is authenticated by the ability to produce a correct response to the challenge, see Fig. 2. Afterwards when the MS is switching between base stations (BSs) within the local MSC area, no authentication is required.

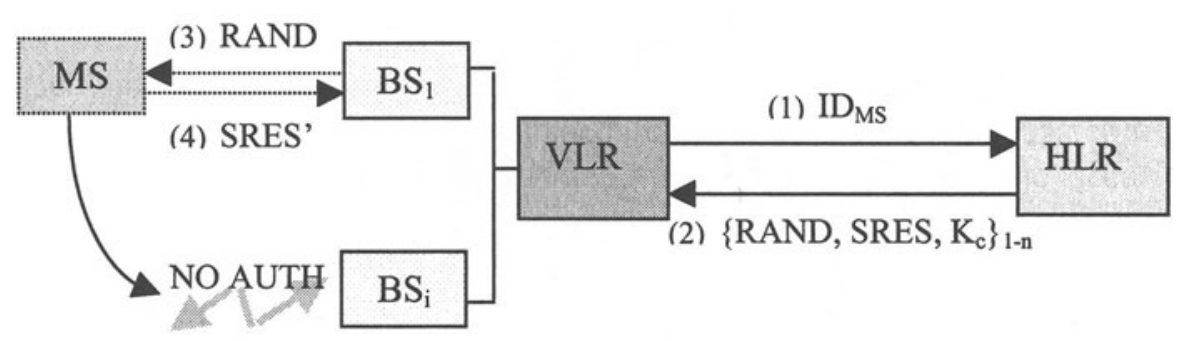

Fig 2. Authentication and handover in GSM

In CDPD mobile units authenticate themselves by presenting a correct historical record (containing an authentication random number and a 
sequence number) which is shared by the mobile and its home domain. Similarly no authentication is required for handover within the same area.

Reuse of established security associations reduces handover latency because no authentication is undertaken at handover. It is possible to achieve smooth, seamless handover that is completely transparent to the users, and the overall system performance can be high.

The drawback of this approach is that skilled impostors might pop up in the middle of a handover and hijack the communication from the real user. If an established security association is represented just by the identity of the mobile terminal, an impostor gains full access by stealing a valid identity. If some kind of secret key is involved in the security association, it is certainly much harder for the impostor to do something meaningful without knowing the key. But as one end of the communication channel changes at handover, the impostor can take advantage of that and launch other attacks. For example he can replay previously intercepted packets by sending them to the new AP again. A widely used replay checking mechanism, the packet sequence number will not help in this case because the new AP does not know those packet sequences that were sent to the previous APs. If other mechanisms, like timestamp or destination address protection, are not feasible on the radio link layer, replay checking has to be done at some higher layer. Besides, in general reusing security associations among many parties leads to more exposure. A single failure may result in security compromises.

\subsection{Re-association}

In this approach, with respect to authentication, a handover is considered no different from the MT's switch-on or its first entrance into a security domain. After a handover the MT must re-associate itself to the new AP as from the beginning. Compared to the approach discussed in the previous section, the security association established here is between a MT and an individual AP rather than between the MT and the current security domain. A representative example of this re-association approach is the IEEE 802.11 wireless LAN [3].

In contrast to GSM and CDPD, authentication is mandatory at handover in IEEE 802.11 wireless LANs. A MT authenticates itself each time it 
connects to a new AP, as Fig. 3 shows. The algorithm to produce a response corresponding to the challenge is WEP (Wired Equivalent Privacy).

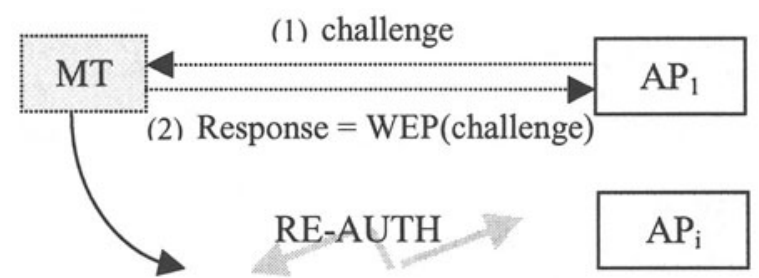

Fig 3. Authentication and handover in IEEE 802.11 Wireless LAN

In consideration of security, this re-association approach is ideal because it leaves no chance for any hijacking at handover. Anyone, including previously authenticated MTs, is rejected unless it can prove its identity again. Because a fresh security association is established after each handover, even if someone got hold of the security attributes he can do mischief only within a short period.

The disadvantage of re-association at each handover is possible high processing overhead and low performance. As stated in the IEEE 802.11 standard WEP is efficient and may be implemented in either hardware or software. Otherwise if the algorithm is complex and computationally intensive, long delays will be experienced at handover. In circumstances where mobile devices are of low or moderate computational power and they undergo frequent handover, this approach is inefficient because the overall system performance can be rather low.

\section{A TOKEN BASED HANDOVER AUTHENTICATION PROTOCOL}

\subsection{Token based approach}

This approach is a trade-off between the security association reuse and re-association approach as described in Section 2.1 and 2.2 respectively. It requires authentication of mobile terminals after each handover, but makes use of the previously established security associations for the purpose of minimising processing overhead. When a MT is going to move, the current AP generates a security token and sends it to the MT. The MT must present 
the correct token in order to be accepted by the destination AP. In this approach the previous AP participates in the handover authentication. It actually vouches to the new AP for the authenticity of a MT that is able to show the right token. In this way the MT's security relationship to the previous AP is moved to the new one, and the MT gets authenticated without undertaking the entire authentication protocol as it did the first time it entered into the security domain. If desired, parts of the security association, for example the encryption key, could be refreshed by using some pre-shared secret to further ensure security.

This approach prevents impostors from hijacking the communication session at handover because they must have the right token in order to be accepted. Compared to the re-association approach, using tokens to transfer security relationships simplifies the handover authentication. It thus reduces the handover latency and makes smooth handover possible.

For mobile users it is usually acceptable to experience some latency when they switch on the device or enter a different area. But in subsequent communication obvious delay or even short signal loss due to handover seems unacceptable. We believe the token based approach to handover authentication allows smooth handover while maintaining security at a reasonable level. Below we present a token based authentication protocol that effectively thwarts handover hijackers without imposing high processing overhead. This protocol has been proposed as one part of the security solution for the HIPERLAN (High Performance Radio Local Area Network) [4] Type 2 wireless LAN.

\subsection{Assumptions}

When a mobile terminal is switched on or it moves into a new security domain, it must successfully go through an entire authentication procedure in order to be accepted. This initial authentication is obviously important, but the detailed protocol for that is not the topic of this paper. From now on we assume that a secure initial authentication protocol is in place. After passing the initial authentication a MT is considered trustworthy, and meanwhile a security association is established between the MT and the connected AP. Because a wireless medium is inherently vulnerable to eavesdropping, we further assume that, as a part of the security association, a shared secret key is arranged for encryption. Only the MT and the AP know this secret key, and it is not an easy job for others to break the key. 


\subsection{Protocol details}

Regardless of different handover types (e.g. mobile initiated, access point initiated, forward handover or backward handover), the currently connected AP can create a security token and send it to the MT before the MT leaves. For the ease of explanation we use the following notations:

$\begin{array}{ll}\boldsymbol{M T}: & \text { mobile terminal } \\ \boldsymbol{I D}_{\boldsymbol{X}}: & \text { X's identifier } \\ \boldsymbol{A P}_{\text {old }}: & \text { access point to which } \mathrm{MT} \text { is connected before handover } \\ \boldsymbol{A P}_{\text {new }}: & \text { access point to which } \mathrm{MT} \text { is connected after handover } \\ \text { Token: } & \text { triplet }\left\{\mathrm{ID}_{\mathrm{MT}}, \text { Nonce, } \mathrm{TS}\right\} \\ \boldsymbol{K}_{\boldsymbol{X}-\boldsymbol{Y}:}: & \text { secret key shared by X and } \mathrm{Y} \\ \boldsymbol{E}(\boldsymbol{K}, \boldsymbol{M}): & \text { encrypt message M with key } \mathrm{K} \\ \boldsymbol{H}(\boldsymbol{K}, \boldsymbol{M}): & \text { compute a hash value over message M with key K } \\ \mathbf{H}(\mathbf{M}): & \text { compute a hash value over message M }\end{array}$

The token in our protocol is a triplet, containing the terminal's identifier ( $\mathrm{ID}_{\mathrm{MT}}$ ), a random value (Nonce), and a timestamp (TS). $\mathrm{ID}_{\mathrm{MT}}$ says for which MT this token is intended. TS shows the time when the token is created. The Nonce and TS together allows effective and efficient replay checking. A token can be used only once and only for the ongoing handover. Therefore its validity time is no longer than the maximum time a handover needs. The $\mathrm{AP}_{\text {new }}$ should always check the arrival time of a handed-over MT against the TS contained in the token. If too much time has elapsed the token must be rejected as invalid. This prevents someone who received the token from holding the token for a long time before using it. Otherwise the token might be misused. When the token is transmitted over the radio link, it is encrypted with $\mathrm{K}_{\mathrm{MT} \text {-APold }}$, the key shared by the MT and the $\mathrm{AP}_{\text {old. }}$. Therefore no one other than the MT can decrypt the token.

\subsubsection{Token transfer between access points via fixed network}

In this alternative the $\mathrm{AP}_{\text {old }}$ sends the token to the $\mathrm{AP}_{\text {new }}$ via the fixed network. The token could be transmitted in plaintext if the fixed network is considered secure, otherwise it could be encrypted with a key shared between access points. If the handover is forward handover, the $\mathrm{AP}_{\text {old }}$ does not know to which access point the MT is moving. Therefore the MT tells the $\mathrm{AP}_{\text {new }}$ about its own identity $\mathrm{ID}_{\mathrm{MT}}$ and the identity of the previous access point $\mathrm{ID}_{\mathrm{APold}}$. The $\mathrm{AP}_{\text {new }}$ then contacts the $\mathrm{AP}_{\text {old }}$ and asks for the security 
token. If the handover is backward, the $\mathrm{AP}_{\text {old }}$ knows to which access point the $\mathrm{MT}$ is moving and thus can send the token to the $\mathrm{AP}_{\text {new }}$ directly. In both cases the MT sends a hash value of the token $H\left(\right.$ Token) to the $\mathrm{AP}_{\text {new }}$. The $\mathrm{AP}_{\text {new }}$ calculates a hash value upon the token obtained from the $\mathrm{AP}_{\text {old }}$ and compares the result to the received $\mathrm{H}$ (Token). If these two hash values match the MT is proved to be authentic. Fig. 4(a) and 4(b) illustrate the authentication procedure in the forward handover and backward handover cases respectively.

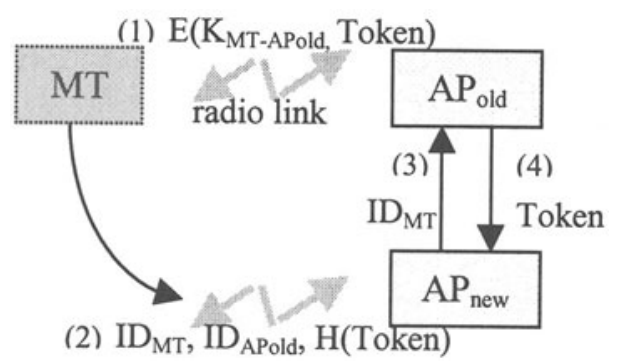

Fig 4(a). Forward handover authentication

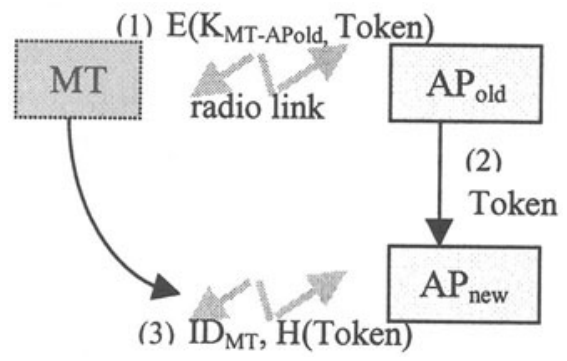

4(b). Backward handover authentication

\subsubsection{Token hash relay via mobile terminal}

This alternative does not use the fixed network to transmit the security token between access points, instead a keyed hash value of the token is first sent to the MT and then relayed by the MT to the $\mathrm{AP}_{\text {new }}$ over the radio link. The purpose of transmitting such a hash value is to prevent the MT from creating a fake token or modifying the token. But a secret key pre-shared by the access points is required to make this keyed hash work.

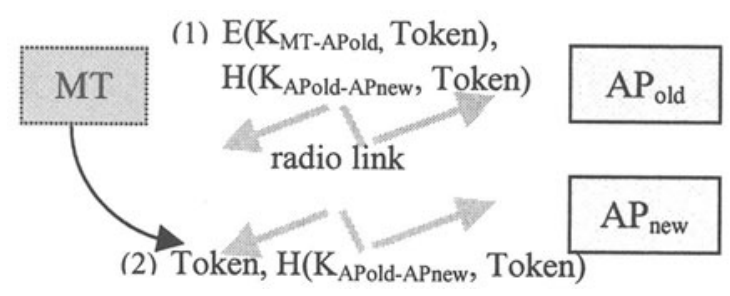

Fig 5. Token hash relay via MT in backward handover

Fig. 5 shows the authentication procedure. First the $\mathrm{AP}_{\text {old }}$ creates a keyed hash value of the token, $\mathrm{H}\left(\mathrm{K}_{\mathrm{APold}-\mathrm{APn} n \mathrm{w}}\right.$, Token $)$, by using the key it shares with the $\mathrm{AP}_{\text {new. }}$. It transmits the hash value to the $\mathrm{MT}$ together with the 
encrypted token. After decrypting the token with key $\mathrm{K}_{\mathrm{MT} \text {-APold }}$ the MT sends the token in plaintext to the $\mathrm{AP}_{\text {new }}$. The hash value from the $\mathrm{AP}_{\text {old }}$ is also forwarded to the $\mathrm{AP}_{\text {new }}$. Upon reception the $\mathrm{AP}_{\text {new }}$ examines the token and calculates a keyed hash value in the same way as the $\mathrm{AP}_{\text {old }}$ did. The result is then compared with the hash value received from the MT. If the hash values equal the $\mathrm{AP}_{\text {new }}$ is sure that the token is from the $\mathrm{AP}_{\text {old }}$ and has not been modified en route. In this way the MT is authenticated.

Because the $\mathrm{AP}_{\text {old }}$ must know to which access point the MT is moving in order to calculate the keyed hash, this alternative is applicable only to backward handovers. If all the access points in the domain share one single key, this alternative could also be used in forward handovers.

\subsubsection{Alternative comparison}

Comparing the token transfer and hash relay alternatives, the former is applicable to both forward handover and backward handover, while the later can be applied only to backward handovers. Because it does not rely on the fixed network for token transfer, the hash relay alternative fits in environments where direct communication between access points is not available or undesired. Besides in terms of the processing overhead for the mobile, the hash relay alternative is more energy saving since only one decryption operation is required. The extra requirement for this alternative is that access points share a secret key for calculating keyed hash.

\subsection{Protocol evaluation}

\subsubsection{Security analysis}

Below we use the BAN Logic [5] to analyse the protocol alternative that relays a keyed hash via the mobile terminal. Security analysis of the other protocol alternative can be done in a similar way.

As illustrated in Fig. 4, the authentication protocol has two messages:

(1) $\mathrm{AP}_{\text {old }} \rightarrow \mathrm{MT}:\left\{\mathrm{E}\left(\mathrm{K}_{\mathrm{MT}-\mathrm{APold}}\right.\right.$, Token $), \mathrm{H}\left(\mathrm{K}_{\mathrm{APold}-\mathrm{APnew}}\right.$, Token $\left.)\right\}$

(2) $\mathrm{MT} \rightarrow \mathrm{AP}_{\text {new }}:\left\{\right.$ Token, $\mathrm{H}\left(\mathrm{K}_{\mathrm{APold}-\mathrm{APnew}}\right.$, Token $\left.)\right\}$ 
At the start of the authentication, we assume that the MT, $\mathrm{AP}_{\text {old }}$, and $\mathrm{AP}_{\text {old }}$ all believe the ownership of shared secret keys. Thus we have
(a) $\mathrm{AP}_{\text {old }}$

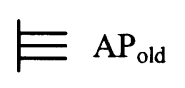
$\stackrel{K_{M T-A P o l d}}{\longrightarrow} \mathrm{MT}$
(b) $\mathrm{MT}$
$\stackrel{\mathrm{K}_{\mathrm{MT}-\mathrm{APol}}}{\longleftrightarrow} \mathrm{AP}_{\text {old }}$
(c) $\mathrm{AP}_{\text {old }}$

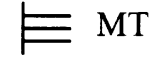
$\stackrel{\mathrm{K}_{\text {APold-APnew }}}{\longleftrightarrow} \mathrm{AP}_{\text {new }}$
(d) $\mathrm{AP}_{\text {new }}$
$\equiv \mathrm{AP}_{\text {old }}$
KaPold-APnosy
$\mathrm{AP}_{\text {old }}$

In message (1) the MT sees $\mathrm{E}\left(\mathrm{K}_{\mathrm{MT}-\mathrm{APold}}\right.$, Token). Because the $\mathrm{MT}$ believes $\mathrm{K}_{\mathrm{MT} \text {-APold }}$ according to assumption (b), by the message meaning rule it believes the $\mathrm{AP}_{\text {old }}$ sent the Token, that is,

$\mathrm{MT} \equiv \mathrm{AP}_{\text {old }} \quad \longrightarrow$ Token

As the Token contains a random value Nonce and a timestamp TS, the MT can check if the Token is fresh. If it is, according to the nonce verification rule, this implies that the MT believes the $\mathrm{AP}_{\text {old }}$ believes the goodness and freshness of the Token.

$$
\mathrm{MT} \equiv \mathrm{AP}_{\text {old }} \equiv \text { Token }
$$

From message (2), assumption (d), and the message meaning rule, we get

$$
\mathrm{AP}_{\text {new }} \equiv \mathrm{AP}_{\text {old }} \quad \sim\left\{\mathrm{H}\left(\mathrm{K}_{\text {APold-APnew }}, \text { Token }\right)\right\}
$$

If the $\mathrm{AP}_{\text {new }}$ calculates a hash value upon the Token received from the MT and the result equals $\mathrm{H}\left(\mathrm{K}_{\mathrm{APold}-\mathrm{AP} \text { ew }}\right.$, Token $)$, the $\mathrm{AP}_{\text {new }}$ believes that the Token is correct, is exactly the one sent by the $\mathrm{AP}_{\text {old }}$. That is,

$$
\mathrm{AP}_{\text {new }} \equiv \mathrm{AP}_{\text {old }} \quad \sim \text { Token }
$$

Since the Token contains a Nonce and a timestamp TS, the $\mathrm{AP}_{\text {new }}$ can check that the Token is fresh. Applying the nonce verification rule we get

$$
\mathrm{AP}_{\text {new }} \equiv \mathrm{AP}_{\text {old }} \rightleftharpoons \text { Token }
$$

And the jurisdiction rule gives

$$
\mathrm{AP}_{\text {new }} \rightleftharpoons \text { Token }
$$

Because only the MT knows the key with which the encrypted token from the $\mathrm{AP}_{\text {old }}$ can be decrypted, the $\mathrm{AP}_{\text {new }}$ believes the sender of the token it received is the right $\mathrm{MT}$. 


\subsubsection{Complexity and performance}

As we can see the handover authentication protocol presented in Section 4.2 is quite simple. It uses a single security token to establish trust. Only two message transmissions are required by the protocol, each containing very few data items. These data items can well be incorporated into the normal handover signalling, without introducing much extra transmission overhead.

The computational demand for mobile terminal is low. What a mobile has to do is to decrypt the token, and to calculate a hash value in the token transfer alternative. Since the token decryption is a secret key operation it is not as time consuming as public key operations. Hash calculations are even faster.

Ericsson's WLAN Guard product uses IKE (Internet Key Exchange) [6] and IPSec [7] to enforce authentication and wireless traffic protection. An entire IKE negotiation (including Diffie-Hellman key exchange, RSA signature creation and verification, HMAC_MD5 hashing, DES encryption, etc.) takes about 3 seconds on an AMD $486100 \mathrm{MHz}$ processor. By reusing established security associations a handover can be completed within 200 milliseconds. If we use the proposed token based authentication protocol (by using DES, MD5 and HMAC_MD5), the handover latency is approximately 400 milliseconds.

\subsubsection{Limitations}

The presented authentication protocol does not help in situations where the security association between a mobile and its connected access point has already been compromised. If an attacker knows the key that is used to protect the security token, it can do handover without problem. This is actually a limitation of the token based authentication approach.

Besides the protocol requires that the current access point generate a token and send it to the mobile just before handover. This might not always be possible. If for some reason the mobile suddenly leaves without notifying the access point, it is impossible for the access point to send any token. In that case the mobile has to re-associate to the new access point. 


\section{CONCLUSION}

In this paper we emphasise the importance of handover authentication and propose a token based authentication approach. This approach compared to others is more attractive with respect to security and handover performance. A token based protocol, in two alternatives, for handover authentication is presented. The protocol plugs the loophole that could be exploited by attackers at handover, while not imposing much processing overhead. With such a handover authentication protocol in place, mobile users can safely migrate and enjoy high system performance.

\section{ACKNOWLEDGEMENTS}

The authors are grateful to Lorens Almehag and Hans Schmekel for their useful suggestions and comments.

\section{REFERENCES}

[1] M. Rahnema, "Overview of the GSM System and Protocol Architecture", IEEE Communications Magazine, Apr. 1993.

[2] CDPD Forum, "Cellular Digital Packet Data System Specification", Release 1.1, Jan. 1995.

[3] IEEE 802.11D3, “Wireless LAN Medium Access Control (MAC) and Physical Layer (PHY) Specifications", Jan. 1996.

[4] ETSI TC-RES, "Radio Equipment and Systems (RES); High Performance Radio Local Area Network (HIPERLAN); Type 1; Functional Specification, Technical Correction”, Dec. 1996.

[5] M. Burrows, M. Abadi, and R. Needham, "A Logic of Authentication", ACM Transactions on Computer Systems, Vol. 8, No. 1, Feb. 1990.

[6] D. Harkins and D. Carrel, "The Internet Key Exchange (IKE)", RFC 2409, November 1998.

[7] S. Kent and R. Atkinson, "Security Architecture for the Internet Protocol", RFC 2401, November 1998. 